\title{
Time and risk perceptions mediate the causal impact of objective delay on delay discounting: An experimental examination of the implicit-risk hypothesis
}

\author{
Jingya Jiang ${ }^{1} \cdot$ Junyi Dai ${ }^{1}$ (D) \\ Accepted: 26 January 2021 / Published online: 25 February 2021 \\ (C) The Psychonomic Society, Inc. 2021
}

\begin{abstract}
Delay discounting refers to the decline in the value of a payoff as the objective delay to its fulfillment increases. Recent research on delay discounting has examined its relationship with time and risk perceptions through correlational studies. Manipulated experiments were conducted in the current research to further investigate the causal links among the relevant variables. Experiment 1 revealed causal influences of objective delay on both risk perception and delay discounting as well as a positive correlation between risk perception and delay discounting. By manipulating risk perception, Experiment 2 demonstrated further a causal impact of risk perception on delay discounting. Experiment 3 manipulated time perception and provided further evidence for causal pathways from time perception to risk perception and delay discounting. Overall, the results verified a causal chain from objective delay to delay discounting through time and risk perceptions in support of the implicit-risk hypothesis regarding delay discounting.
\end{abstract}

Keywords Delay discounting $\cdot$ Risk perception $\cdot$ Time perception $\cdot$ Causality $\cdot$ Implicit risk hypothesis

\section{Introduction}

Many everyday decisions involve tradeoffs between outcomes occurring at different times. In such intertemporal choices, people tend to prefer an immediate payoff against a delayed one with the same amount. This phenomenon is called delay discounting, as the value of the payoff declines as its fulfillment is delayed into the future (Frederick, Loewenstein, \& O'Donoghue, 2002).

Psychological mechanisms underlying delay discounting have been a central topic in the research on intertemporal choice. As a pioneer researcher, John Rae once suggested that delay might lead to a failure in harvesting the anticipated benefit, and the uncertainty of one's life situation plays a crucial role in time preference (Rae, 1834). Many contemporary scholars have made similar arguments (e.g., Blackburn \& ElDeredy, 2013; Read, 2004; Takahashi, Ikeda, \& Hasegawa,

Junyi Dai

junyidai@zju.edu.cn

1 Department of Psychology and Behavioral Sciences, Zhejiang University, No. 148 Tianmushan Road, Hangzhou 310028, China
2007). For instance, Prelec and Loewenstein (1991) deemed uncertainty an inevitable feature of anything delayed. Similarly, Rachlin, Raineri, and Cross (1991) argued that one of the reasons people prefer a smaller-but-sooner (SS) reward over a larger-but-later (LL) reward is that nowadays it is unlikely that people would receive the promised delayed reward. Some researchers further suggested that the uncertainty implied by delay was a fundamental, if not the only, reason for delay discounting (e.g., Sozou, 1998; Epper, Fehr-Duda, \& Bruhin, 2011). Specifically, the longer the delay is, the more likely that something unexpected would occur during the delay period to eliminate the anticipated benefit. The increasing level of risk would make the delayed reward less and less attractive - that is, delay discounting (i.e., the implicit-risk hypothesis; e.g., Benzion, Rapoport, \& Yagil, 1989; Keren \& Roelofsma, 1995; Saito, 2009; Weber \& Chapman, 2005). This hypothesis might be interpreted as a single-process theory for delay and probability discounting, which suggests time and risk preferences arise from a single process. Such a strong interpretation of the hypothesis, however, is not supported by empirical research (e.g., Du, Green, Myerson, 2002; Weber \& Huettel, 2008). See Johnson, Bixter, and Luhmann (2020) for a recent review and a meta-analytic study on the issue, and 
Dai, Pachur, Pleskac, and Hertwig (2019) for a systematic analysis of the role of risk and uncertainty in intertemporal choice.

The implicit-risk hypothesis has enjoyed considerable support from animal studies (e.g., Kagel, Green, \& Caraco, 1986; Stephens, 2002; Wendt \& Czaczkes, 2017), which suggested a causal link from risk perception to delay discounting. Does the same link also underlie the delay discounting phenomenon in human time preference? Several empirical studies have attempted to address this question. For example, Patak and Reynolds (2007) asked participants to perform a delaydiscounting task and then estimate the risk of not receiving the anticipated future reward. The results showed a moderately positive correlation between the rating of uncertainty and the rate of delay discounting. Consequently, it was suggested that participants incorporated their estimates of uncertainty inherent in delayed rewards while evaluating the options for the delay-discounting task.

The above implication is certainly insightful but not very convincing given several drawbacks of the reported study. First, it was inferred from correlational instead of causal data that participants viewed delayed reward as risky reward and the higher risk associated with a longer delay led to apparently higher rate of delay discounting. Second, participants in the study were asked whether they were sure of receiving the $\$ 10$ standard delayed by a certain period before reporting how sure they were. The first question might serve as a subtle cue (i.e., a demand characteristic) that triggered participants to consciously consider the risk only in the risk-perception task but not in the delay-discounting task, or even prepared participants to report a higher level of uncertainty given a longer delay for the second question. Similar impacts might also occur unconsciously, resulting in a priming effect. Third, participants were asked to imagine that they had chosen the delayed standard while reporting their risk perception. Consequently, their responses might be influenced by their choices to maintain cognitive consonance. Specifically, participants might report a lower degree of uncertainty for the delayed option to justify their choice of the option. Therefore, it was unclear whether the level of uncertainty they reported was consistent with the risk they perceived as they performed the delay-discounting task. Finally, because risk perception was measured after the delay-discounting task, the reported level of uncertainty might suffer from an order effect, making it an inappropriate measure of the actual level of risk perception underlying delay discounting.

Similarly, Bixter and Luhmann (2015) explored whether risk was a major reason for delay discounting. Specifically, they examined whether the order of presenting delay and probability information would affect participants' preference between rewards involving both delay and risk. It was found that participants were more likely to prefer LL rewards and responded faster when the delay information was presented before probability information than the other way around. These results were interpreted as if participants would transform delay into risk and presenting delay information first facilitated the processing of the subsequent probability information. This interpretation was in turn used as evidence for the implicit-risk hypothesis. However, since the reported studies had not directly manipulated participants' risk perception, the shorter response time when the probability information was presented secondly provided only weak evidence for the argument that delay was transformed into risk and could not verify a causal link from implicit risk to delay discounting. Moreover, the evidence supporting the implicit-risk hypothesis was mainly obtained from a task with options involving explicit delay and risk simultaneously. Such options differed from the common type of stimuli in delay-discounting studies, that is, options with only known payoff and delay, but not any explicit information on risk or uncertainty. Consequently, more direct and stronger evidence for the implicit-risk hypothesis is still needed.

Like risk perception, time perception is another significant psychological factor for time preference. Zauberman, Kim, Malkoc, and Bettman (2009) showed that subjective delay was a nonlinear and concave function of objective delay, consistent with the general psychophysical principle. Additionally, their studies demonstrated that the nonlinear mapping of objective delay to subjective delay is an important factor underlying the hyperbolic pattern of delay discounting. Similarly, Read, Frederick, Orsel, and Rahman (2005) found a robust anomaly in intertemporal choice - that is, the date/ delay effect that suggests discount rate tends to be lower when a delay is described in terms of the due calendar date (e.g., 1 May) than when it is described as the length of delay (e.g., in 6 months). It appeared that time perception was longer when a delay was described as a duration than when it was described in terms of the corresponding calendar date. The results suggested that it was subjective rather than objective delay that directly influenced the degree of delay discounting and thus time preference (see also LeBoeuf, 2006).

In summary, previous studies have revealed two crucial psychological factors underlying delay discounting, that is, time and risk perceptions. However, the relevant research provided only correlational or indirect evidence for a causal chain from objective delay to delay discounting. For a deeper understanding of the psychological mechanism underlying delay discounting, we conducted three manipulated experiments to shed new light on the causal chain.

\section{Experiment 1}

The purpose of this study was twofold: (1) to demonstrate a causal impact of objective delay on risk perception and delay discounting, and (2) to determine a proper measurement order 
of risk perception and delay discounting to obtain a more appropriate, uncontaminated measure of each variable. The causal relationships revealed in this study would constitute the first pieces of evidence for a causal pathway from objective delay to delay discounting, and the proper measurement order would be adopted in subsequent studies for a more legitimate investigation of the relationships among the interconnected factors.

\section{Method}

Participants Eighty-six students (55 females, $\mathrm{M}_{\mathrm{age}}=20.22$ years, $\mathrm{SD}_{\text {age }}=3.16$ years) from a top university in China were recruited for the study. One participant was dropped from data analysis due to misunderstanding the requirements of the study. The remaining participants were randomly assigned into two groups. The first group consisted of 41 participants (28 females, $\mathrm{M}_{\text {age }}=20.71$ years, $\mathrm{SD}_{\text {age }}=4.13$ years) who completed the delay-discounting task before the riskperception task, whereas the second group consisted of 44 participants (27 females, $\mathrm{M}_{\mathrm{age}}=19.77$ years, $\mathrm{SD}_{\mathrm{age}}=1.72$ years) who completed the delay-discounting task after the risk-perception task. Hereafter the first group is referred to as the delay-first group and the second group as the risk-first group.

Materials and procedure The experiment was introduced to the participants as a study on their preferences between different payment plans. Specifically, participants were required to show their preferences between immediate receipt of smaller amounts of money and delayed receipt of larger amounts of money. They were also told that their payment for participation would be determined by their response to one randomly selected choice question in the delay-discounting task. At the end of the experiment, each participant could randomly select a trial from the delay-discounting task and would be paid according to the actual choice in that trial. If the immediate option was chosen, the participant would be paid the amount right after the experiment. If the delayed option was chosen, the participant would receive the payment at the specified delay time. Demographic information (e.g., gender and age) was also collected before the experiment started.

A titration procedure was adopted for the delaydiscounting task. Specifically, in each trial participants needed to choose between a larger reward (i.e., 40 Chinese Yuan (CNY)) after a certain delay $(1,7,30,180$, or 365 days) and a smaller but immediate reward. The amount of the immediate reward would be adjusted upwards if participants chose the delayed reward and downwards if participants chose the immediate reward. For each delay, the titration procedure continued until the immediate amount fell with a narrow range of possible values, which would in turn be used to determine the indifference point for the specific delay. See the Appendix for details of the titration procedure.

In the risk-perception task, participants were required to report their perceived probability of receiving the delayed 40 CNY if they needed to choose between some immediate money and the delayed reward. A sliding bar was shown on the computer screen together with the instruction, and participants were to adjust the bar to indicate their perceived probability of receiving the delayed reward as a measure of perceived risk. Compared with the instruction used in Patak and Reynolds (2007), the current instruction could arguably lessen the impacts of demand characteristic, priming effect, and the need for cognitive consonance on risk perception. This was because participants in the current study were neither asked whether they were sure of receiving the delayed reward nor required to respond while imagining they had chosen the delayed reward.

Data analysis Individual indifference points obtained from the delay-discounting task were first examined with the nonsystematic data screening criteria for delay discounting proposed by Rung, Argyle, Siri, and Madden (2018). A set of individual data was considered nonsystematic if the indifference point for any specific delay was greater than the indifference point for the preceding (and thus shorter) delay in the sequence of tested delays (i.e., 1, 7, 30, 180, or 365 days) by at least $20 \%$ of the amount of the LL reward, or the indifference point for the longest delay was not lower than the indifference point for the shortest delay by at least $10 \%$ of the amount of the LL reward. Data from any participants whose indifference points in the delay-discounting task failed the criteria were excluded from further analysis.

Overall levels of risk perception and delay discounting for each participant were measured in terms of area under the curve (AUC; Myerson, Green, \& Warusawitharana, 2001). To calculate AUC, we first transformed the objective delays, perceived probabilities, and indifference points for each participant into corresponding proportions against the longest delay (i.e., 365 days), the highest perceived probability (i.e., $100 \%$ ), and the largest possible indifference points (i.e., 40 CNY), respectively. These normalized values were then used as the horizontal and vertical ordinates to construct the curve and calculate the related AUC. The value of AUC was constrained between 0 and 1, with larger values representing less delay discounting and lower risk perception.

When associations between variables were of concern, Kendall's tau was used as a measure of correlation since the relationship was likely to be monotonic but not linear. Specifically, we examined the associations between objective delay, perceived probability, and corresponding discount factor (i.e., the ratio of indifference point to the amount of delayed reward) to investigate causal or correlational relationships among the relevant variables. These analyses were performed both at a group level when data from individual 
participants were pooled together and at an individual level when the correlation coefficients were calculated for each participant. Note that lower values of perceived probability and discount factor indicated higher levels of risk perception and delay discounting. When group differences in AUC were of concern, Mann-Whitney U tests were adopted since the AUC measure was better treated as an ordinal variable rather than an interval variable. We also analyzed the association between overall levels of risk perception and delay discounting (as measured by relevant AUCs) across participants.

All analyses in this and the following studies were performed using a Bayesian approach with the JASP software (JASP Team, 2019) and Mplus software (Muthén \& Muthén, 1998-2017). The Bayesian approach could offer supporting evidence for either the null or the alternative hypothesis (e.g., Rouder, Speckman, Sun, \& Morey, 2009). Specifically, the Bayes factor (BF) between two hypotheses shows the relative amount of supporting evidence from the data for one hypothesis against the other; when the BF of the null hypothesis against the alternative hypothesis (i.e., $\mathrm{BF}_{01}$ ) differs from 1 substantially (i.e., $>3$ or $<1 / 3$, Jeffery, 1961), one can make a statistical inference to accept the better hypothesis. Statistical inference can also be made based on the 95\% credible interval (CI) of the relevant model parameter, which shows a range of credible values that has a 95\% probability of covering the true value of the parameter (Kruschke \& Liddell, 2018). When such intervals do not include the null value, one can make a statistical inference to accept the alternative hypothesis (Lindley, 1965). In this paper, we combined the above two criteria to make statistical inferences.

\section{Results}

Five participants (three in the delay-first group and two in the risk-first group) did not pass the systematic screening. Consequently, all the analyses reported below were based on the data from the remaining 80 participants ( 38 in the delayfirst group and 42 participants in the risk-first group). ${ }^{1}$

Figure 1 shows the scatterplots for the group-level associations among objective delay, perceived probability, and discount factor for the delay-first and risk-first groups separately, and Table 1 shows the results of the corresponding correlation analyses. For both groups, objective delay was negatively correlated with perceived probability and discount factor, and perceived probability was positively correlated with discount factor. The same pattern also occurred with each participant since each individual coefficient had the same sign as the group coefficient. See Fig. 2 for the distribution of individual Kendall's tau for each association.

\footnotetext{
${ }^{1}$ The datasets generated and/or analyzed during this and the following studies are available at https://osf.io/q96en/.
}

Table 2 shows the results of the Mann-Whitney U tests for the differences in the overall levels of delay discounting and risk perception between the delay-first and risk-first groups. For the difference in delay-discounting level, the BF and 95\% CI suggested negligible evidence for either hypothesis. On the other hand, both the BF and the $95 \% \mathrm{CI}$ for the difference in risk perception favored the null hypothesis. Finally, the Bayesian correlation analysis showed a positive association between the overall levels of risk perception and delay discounting across participants (Kendall's tau $=0.295, \mathrm{BF}_{10}$ $=241.013,95 \% \mathrm{CI}=[0.140,0.429])$, suggesting that people who perceived a higher level of risk also tended to discount future payoffs more heavily.

\section{Discussion}

By manipulating objective delays and measuring the resultant levels of delay discounting (i.e., discount factor) and risk perception (i.e., perceived probability), this study provided clear evidence for a causal impact of objective delay on risky perception and delay discounting at both group and individual levels. As expected, longer objective delays led to higher levels of both delay discounting and risk perception. Furthermore, levels of delay discounting and risk perception were positively correlated at both group and individual levels, and more importantly, measurement order did not change the nature of associations among objective delay, risk perception, and delay discounting. Finally, there was clear evidence that the measurement of delay discounting did not impact the results of the subsequent measurement of risk perception, justifying the measurement order adopted in Patak and Reynolds (2007).

Besides explicitly testing the influence of measurement order to verify the approach of previous studies (Patak \& Reynolds, 2007; Reynolds, Patak, \& Shroff, 2007), the relevant instruction was modified in this study to lessen the impacts of demand characteristic, priming effect, and the need for cognitive consonance on participants' responses to the risk-perception task. Therefore, the current results not only corroborated those from previous research, but also extended them in a context more appropriate for measuring risk perception underlying delay discounting. However, as with Patak and Reynolds, this study provided only correlational evidence for the association between risk perception and delay discounting. Therefore, a second experiment was conducted to address this issue for more direct evidence for the implicitrisk hypothesis.

\section{Experiment 2}

The major goal of this experiment was to establish a causal link from risk perception to delay discounting. Specifically, 

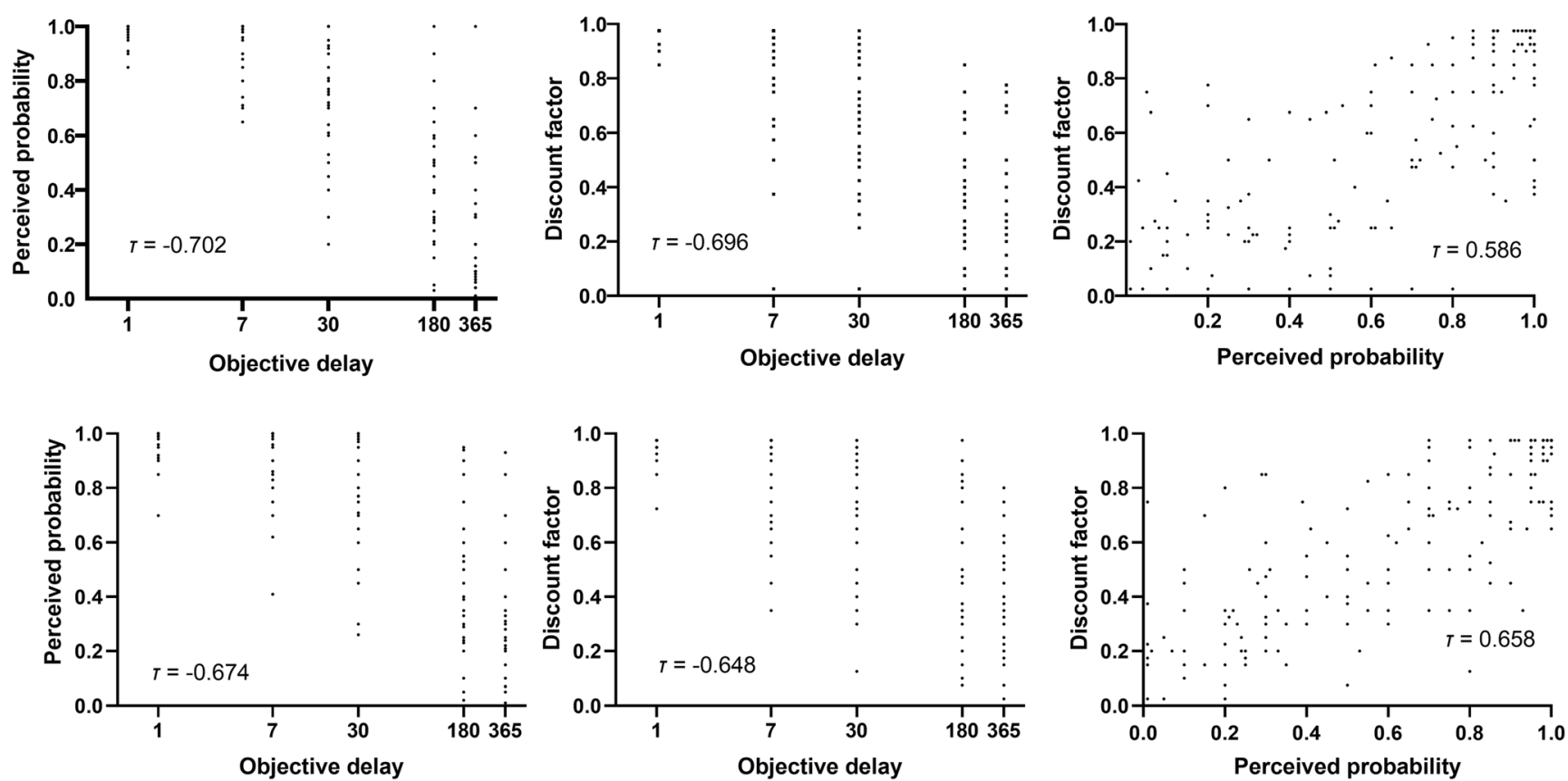

Fig. 1 Scatterplots for the group-level associations among objective delay, perceived probability, and discount factor for the delay-first group (upper panels) and risk-first group (lower panels) in Experiment 1. The axis for objective delay was logarithmic-transformed to express the values more clearly

participants' risk perception was directly manipulated to examine whether it would influence the subsequent level of delay discounting.

\section{Method}

Participants Seventy-four students (48 females, $\mathrm{M}_{\mathrm{age}}=18.64$ years, $\mathrm{SD}_{\mathrm{age}}=1.09$ years) from a top university in China were recruited for this study. The participants were randomly assigned into two groups: a high-risk group with 38 participants (26 females, $\mathrm{M}_{\mathrm{age}}=18.60$ years, $\left.\mathrm{SD}_{\mathrm{age}}=0.99\right)$ and a low-risk group with 36 participants (22 females, $\mathrm{M}_{\text {age }}=18.67$ years, $\mathrm{SD}_{\text {age }}=1.18$ years).

Materials and procedure Materials and procedure of this study were similar to those of Experiment 1 with three changes. First, participants in the low-risk group received the same instruction regarding the payment method as in Experiment 1, whereas participants in the high-risk group were told to remind the experimenter of the payment on its due date if they chose the LL option in the randomly selected trial. Second, we adjusted delay lengths so that none of the due dates would be on a holiday. This consideration led to the following delays used in the current study: 1, 7, 15, 30, 120, 180, and 365 days. Third, as suggested by the results of Experiment 1, each participant completed the delay-discounting task before the riskperception task to obtain an uncontaminated measure of each variable.

Data analysis The same data-screening procedure and analyses were conducted in this study as in Experiment 1. To provide further evidence for the implicit-risk hypothesis, we also ran Bayesian path analyses to examine the direct and indirect (via risk perception) effects of objective delay on delay discounting with Mplus 8.0.

\section{Results}

Fifteen participants (seven in the low-risk group and eight in the high-risk group) were dropped from further analyses

Table 1 Results of group-level Bayesian correlation analyses in Experiment 1

\begin{tabular}{llll}
\hline & Objective delay & Perceived probability & Discount factor \\
\hline Objective delay & & $3.606 \times 10^{43}[-0.784,-0.595]$ & $5.773 \times 10^{42}[-0.778,-0.589]$ \\
Perceived probability & $3.142 \times 10^{44}[-0.754,-0.575]$ & & $1.105 \times 10^{30}[0.481,0.669]$ \\
Discount factor & $1.232 \times 10^{41}[-0.727,-0.549]$ & $2.653 \times 10^{42}[0.557,0.735]$ & \\
\hline
\end{tabular}

Note. Each cell shows the Bayes factor in favor of the alternative hypothesis and the 95\% CI of the Kendall correlation coefficient (in brackets). The results of the delay-first group are shown above the diagonal whereas those of the risk-first group are shown below the diagonal 

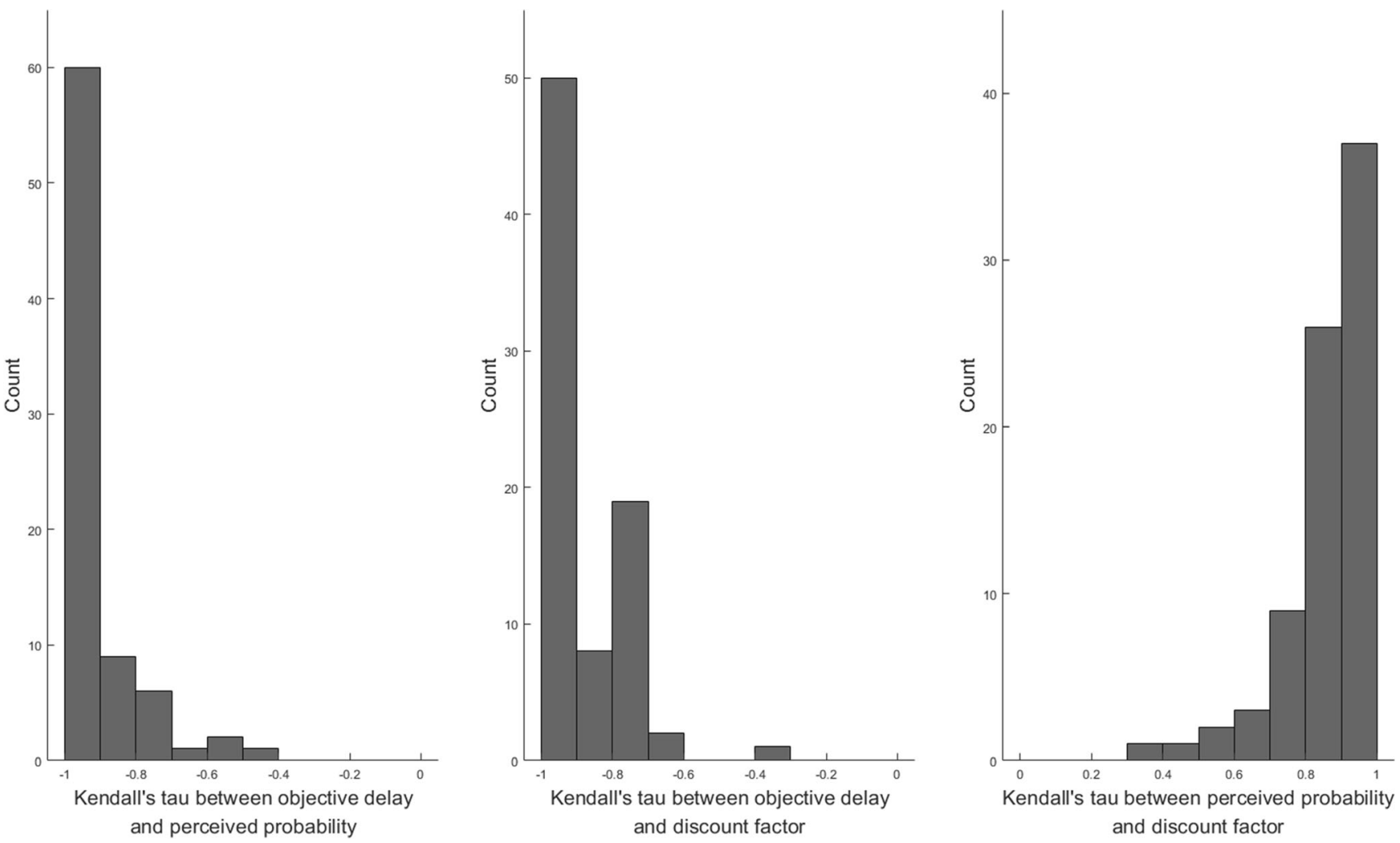

Fig. 2 Distributions of individual Kendall's tau for the associations among objective delay, perceived probability, and discount factor in Experiment 1

because of a failure to pass the systematic screening. Consequently, the analyses reported below were based on 59 remaining participants ( 29 in the low-risk group and 30 in the high-risk group).

Table 3 shows the results of the Mann-Whitney U tests on the differences in risk perception and delay discounting levels between the two groups. The participants in the low-risk group perceived a lower level of risk on average than the high-risk group, verifying the validity of the experimental manipulation. As expected, the low-risk group also showed a lower level of delay discounting than the high-risk group on average. As in Experiment 1, the results of Bayesian correlation analyses suggested that there was an association between the overall levels of risk perception and delay discounting across all participants (Kendall's tau $=0.445, \mathrm{BF}_{10}=$ $\left.3.15 \times 10^{4}, 95 \% \mathrm{CI}=[0.255,0.589]\right)$. The same was true when participants from either group were analyzed separately (for the low-risk group, Kendall's tau $=0.305, \mathrm{BF}_{10}=3.217,95 \%$
$\mathrm{CI}=[0.039,0.512] ;$ for the high-risk group, Kendall's tau = $\left.0.329, \mathrm{BF}_{10}=5.384,95 \% \mathrm{CI}=[0.065,0.530]\right)$.

Table 4 shows the results of group-level correlation analyses among objective delay, perceived probability, and discount factor for each group, and Fig. 3 shows the relevant scatterplots. Again, objective delay was negatively correlated with perceived probability and discount factor, and perceived probability was positively correlated with discount factor. The same pattern also occurred in almost each participant (see Fig. 4). Finally, Bayesian path analyses revealed evidence for both the direct effect of objective delay on delay discounting and the indirect effect via risk perception. See Table 5 for the point estimates and $95 \%$ CIs of the standardized path coefficients.

\section{Discussion}

By experimentally manipulating participants' risk perception, this study found direct evidence for a causal impact of risk
Table 2 Results of Bayesian Mann-Whitney U tests on AUC measures for the differences in the levels of delay discounting and risk perception between the delay-first and risk-first groups in Experiment 1

\begin{tabular}{lllll}
\hline & Mean of DF group & Mean of RF group & $\mathrm{BF}_{10}$ & $\begin{array}{l}95 \% \text { credible intervals } \\
\text { of effect size }\end{array}$ \\
\hline Delay discounting & 0.402 & 0.486 & 1.139 & {$[-0.812,0.054]$} \\
Risk perception & 0.473 & 0.491 & 0.251 & {$[-0.475,0.342]$} \\
\hline
\end{tabular}

$D F$ delay-first, $R F$ risk-first 
Table 3 Results of Bayesian Mann-Whitney U tests on AUC measures for the differences in the levels of risk perception and delay discounting between the low-risk and high-risk groups in

Experiment 2

\begin{tabular}{lllll}
\hline & $\begin{array}{l}\text { Mean of } \\
\text { low-risk group }\end{array}$ & Mean of high-risk group & $\mathrm{BF}_{10}$ & $\begin{array}{l}\text { 95\% credible intervals } \\
\text { of effect size }\end{array}$ \\
\hline Risk perception & 0.563 & 0.339 & 21.198 & {$[0.254,1.338]$} \\
Delay discounting & 0.658 & 0.370 & 180.889 & {$[0.437,1.576]$} \\
\hline
\end{tabular}

perception on delay discounting. When participants were responsible for reminding the experimenter for a delayed payment, they perceived higher risk of missing the payment. Consequently, they appeared to discount the value of a delayed payment more heavily than participants in the low-risk group who did not carry the burden of reminding the experimenter. This clearly supported the implicit-risk hypothesis, which suggests that, while evaluating a delayed outcome, people would consider the associated risk of missing the outcome. The results of relevant group- and individual-level correlation analyses and the credible indirect effect from objective delay to delay discounting via risk perception were also consistent with this causal interpretation. Although previous studies (e.g., Benzion, Rapoport, \& Yagil, 1989; Bixter \& Luhmann, 2015; Stevenson, 1986) have suggested such a causal link, they provided only indirect evidence for the relevant hypothesis. Finally, the credible direct effect from objective delay to delay discounting suggested that risk perception was not the only reason for delay discounting, echoing the existing evidence against the strong interpretation of the implicit-risk hypothesis as a single-process theory of delay and probability discounting.

Combined, the experiments reported so far suggested a causal chain from objective delay to delay discounting through risk perception. A few studies also suggested a critical role of time perception in the construction of intertemporal preferences. However, like studies on the relationship between risk perception and delay discounting, most studies on the relationship between time perception and delay discounting were also correlational. Therefore, a third experiment was conducted to establish a causal role of time perception in delay discounting.

\section{Experiment 3}

The major goal of this experiment was to establish a causal link from objective delay to risk perception through (subjective) time perception. Specifically, participants' time perception was directly manipulated using the date/delay effect (Read et al., 2005) to examine whether it serves as a causal mediator between objective delay and risk perception.

\section{Method}

Participants One-hundred and thirty-nine students from a top university in China were recruited for this study. Seven participants were dropped from further analysis due to misunderstanding the study requirements or misreading the delays. The remaining participants were randomly assigned into two groups: a short-perception group with 64 participants (50 females, $\mathrm{M}_{\mathrm{age}}=20.44$ years old, $\mathrm{SD}_{\mathrm{age}}=2.07$ years $)$ and a longperception group with 68 participants $\left(54\right.$ females, $M_{\text {age }}=$ 20.10 years, $\mathrm{SD}_{\text {age }}=1.84$ years $)$.

Materials and procedure Previous studies (Dehart \& Odum, 2015; LeBoeuf, 2006; Read et al., 2005) had demonstrated that a given delay led to a lower level of delay discounting when it was shown in terms of the due calendar date than when it was shown in terms of the length of delay. For example, a delay of 15 days from 1 January 2020 can be shown as either being 15 days or on 16 Jan 2020; the former tends to produce more delay discounting than the latter. One possible explanation for the above phenomenon was that different presentation modes of objective delays led to different time perceptions and in turn distinct levels of delay discounting. Therefore, in this study the same delays were shown as due dates for participants in the short-perception group, but as the lengths of delay for participants in the long-perception group to manipulate time perception and examine whether time perception was a causal factor for risk perception and delay discounting.

Participants in the current study were required to complete three successive tasks, a delay-discounting task, a risk-

Table 4 Results of group-level Bayesian correlation analyses in Experiment 2

\begin{tabular}{llll}
\hline & Objective delay & Perceived probability & Discount factor \\
\hline Objective delay & & $9.189 \times 10^{23}[-0.593,-0.411]$ & $4.950 \times 10^{42}[-0.752,-0.571]$ \\
Perceived probability & $1.228 \times 10^{47}[-0.772,-0.593]$ & & $1.988 \times 10^{24}[0.411,0.593]$ \\
Discount factor & $9.773 \times 10^{39}[-0.719,-0.541]$ & $5.875 \times 10^{36}[0.513,0.693]$ & \\
\hline
\end{tabular}

Note. Each cell shows the Bayes factor in favor of the alternative hypothesis and the $95 \%$ CI of the Kendall correlation coefficient (in brackets). The results of the low-risk group are shown above the diagonal, whereas those of the high-risk group are shown below the diagonal 

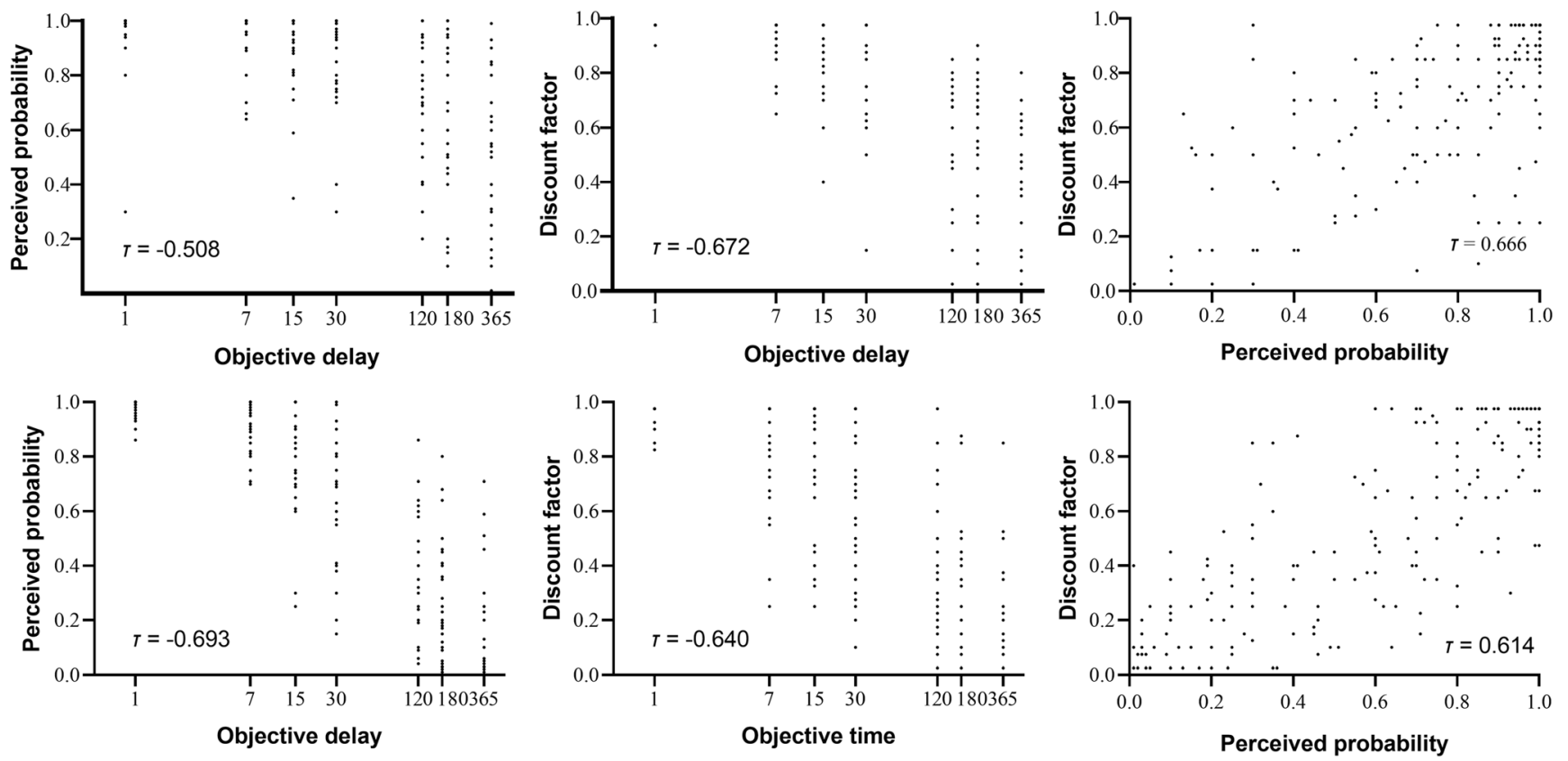

Fig. 3 Scatterplots for the group-level associations among objective delay, perceived probability, and discount factor for the low-risk group (upper panels) and high-risk group (lower panels) in Experiment 2. The axis for objective delay was logarithmic-transformed to express the values more clearly

perception task, and a time-perception task. The first two tasks were similar to those in the previous experiments except for the manipulation of presentation modes of objective delays. In addition, delays of $1,7,15,30,60,180$, and 365 days were used in the current study to avoid any due date falling on a holiday. The delay-discounting task was performed under a high-risk condition, that is, all participants were required to remind the experimenter of the payment on its due date if they chose the LL option in the randomly selected trial.
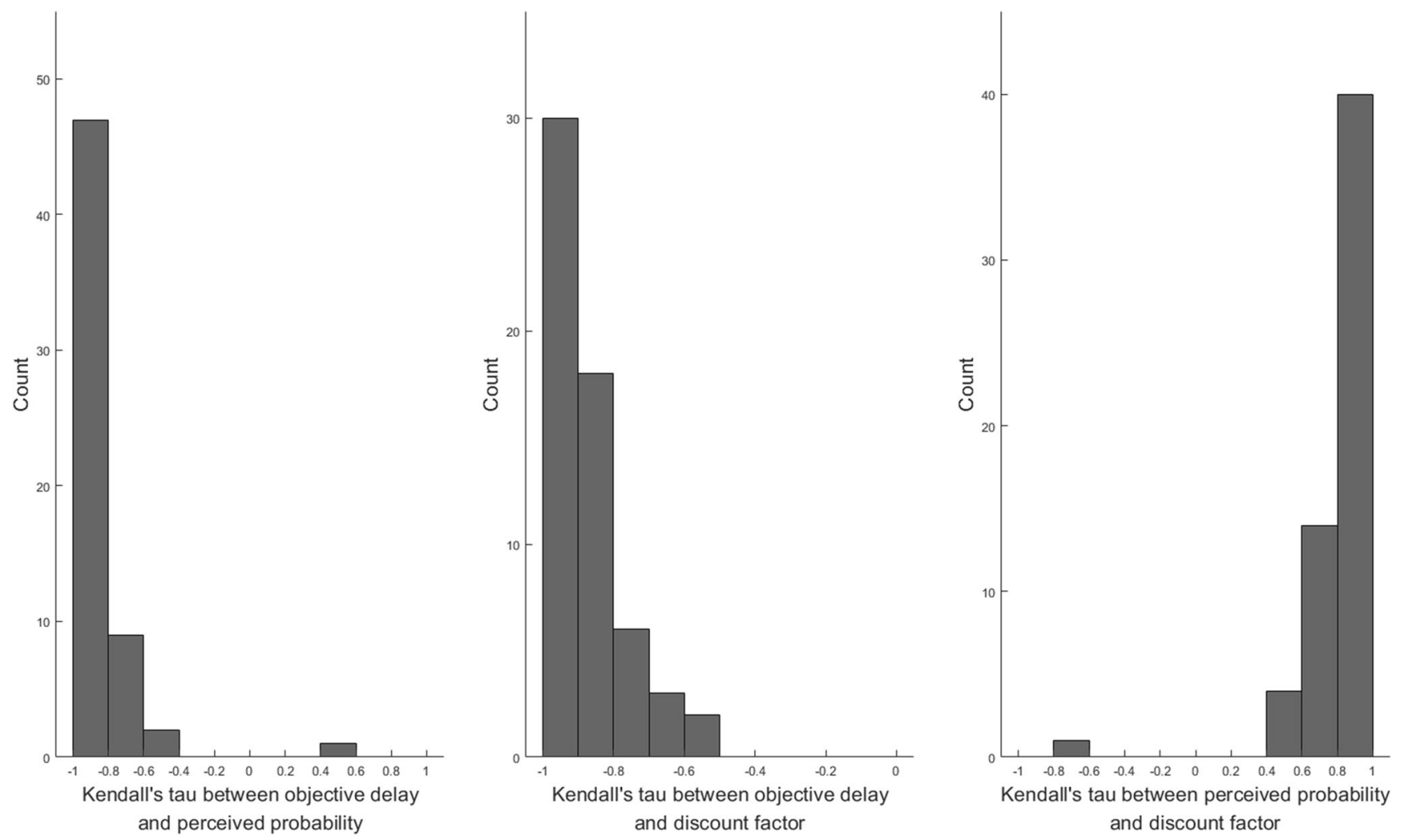

Fig. 4 Distributions of individual Kendall's tau for the associations among objective delay, perceived probability, and discount factor in Experiment 2 
Table 5 Point estimates and 95\% CIs (in brackets) of the path coefficients from the Bayesian path analyses regarding the direct and indirect (via risk perception) effects of objective delay on delay discounting in Experiment 2

\begin{tabular}{lllr}
\hline Effect & Low-risk group & High-risk group & Across \\
\hline Direct & $-0.604[-0.731,-0.477]$ & $-0.406[-0.545,-0.266]$ & $-0.421[-0.510,-0.330]$ \\
Indirect & $-0.080[-0.160,-0.005]$ & $-0.291[-0.403,-0.185]$ & $-0.242[-0.308,-0.180]$ \\
\hline
\end{tabular}

In the time-perception task, the participants were shown a scale on which they needed to rate how long they thought a certain delay was by moving a slider to the corresponding position. The two ends of the scale were marked with labels of "very short" and "very long" as in previous studies on time perception (e.g., Zauberman, et al., 2009). Suppose the experiment was performed on 1 January 2020 and the time perception of a 15-day delay was to be measured. Then participants in the long-perception group were asked to rate how long he/ she thought a delay of 15 days was, whereas those in the shortperception group were asked to rate how long he/she thought the time interval between today and 16 January 2020 was.

Data analysis As in the previous two studies, individual indifference points were first screened to find and remove participants who appeared to respond in an irregular manner in the delay-discounting task. After that, we used AUC to measure the overall level of time perception as well as delay discounting and risk perception as in previous studies. To calculate AUC measures for time perception, for each objective delay, we first transformed the distance of the slider from the leftmost position of the scale into its ratio to the maximum possible distance (i.e., the length of the scale) as a measure of time perception for the objective delay. Such proportions were then combined with normalized objective delays (i.e., the ratio of objective delays to the longest objective delay) to construct a curve and calculate the related AUC for each participant. We also ran the same major analyses as in the previous two studies, and several Bayesian path analyses to examine the direct and indirect (via time perception) effects of objective delay on risk perception.

\section{Results}

Twenty-four participants (ten in the short- perception group and 14 in the long-perception group) were dropped from further analyses due to a failure to pass the screening test. Consequently, the follow-up results were based on the remaining 108 participants (54 in either group).

Table 6 shows the results of the Mann-Whitney U tests regarding the differences between the two groups in time perception, risk perception, and delay-discounting levels measured by AUC. Participants in the short-perception group tended to perceive the same set of objective delays as being shorter than those in the long-perception group, verifying the validity of the experimental manipulation. In addition, the two groups differed in the levels of risk perception and delay discounting given the same set of objective delays: Participants in the short-perception group on average perceived a lower level of risk and demonstrated a lower level of delay discounting than those in the long-perception group. The results of Bayesian correlation analyses revealed a positive association between the overall levels of risk perception and delay discounting across all participants (Kendall's tau = $\left.0.444, \mathrm{BF}_{10}=1.12 \times 10^{9}, 95 \% \mathrm{CI}=[0.306,0.556]\right)$. The same was true when participants from either group were analyzed separately (for the short-perception group, Kendall's tau = $0.379, \mathrm{BF}_{10}=542.264,95 \% \mathrm{CI}=[0.184,0.534]$; for the long-perception group, Kendall's tau $=0.472, \mathrm{BF}_{10}=$ $\left.4.28 \times 10^{4}, 95 \% \mathrm{CI}=[0.270,0.619]\right)$. Note that larger values of AUC regarding risk perception and delay discounting indicated lower levels of the respective variables, whereas larger values of AUC regarding time perception represented higher levels of the target variable.

Table 7 shows the results of group-level correlation analyses for objective delay, subjective delay, perceived probability, and discount factor for each group. Objective delay was again negatively correlated with perceived probability and discount factor, and perceived probability was positively correlated with discount factor. In addition, subjective delay was positively correlated with objective delay and negatively correlated with perceived probability and discount factor. See
Table 6 Results of Bayesian Mann-Whitney U tests on AUC measures for the group differences in the levels of time perception, risk perception, and delay discounting in Experiment 3

\begin{tabular}{lllll}
\hline & Mean of SP group & Mean of LP group & $\mathrm{BF}_{10}$ & $\begin{array}{l}95 \% \text { credible intervals } \\
\text { of effect size }\end{array}$ \\
\hline Time perception & 0.579 & 0.733 & 2394.027 & {$[-1.414,-0.612]$} \\
Risk perception & 0.573 & 0.469 & 3.293 & {$[0.071,0.838]$} \\
Delay discounting & 0.565 & 0.452 & 3.111 & {$[0.069,0.813]$} \\
\hline
\end{tabular}

$S P$ short-perception, $L P$ long-perception 
Table 7 Results of group-level Bayesian Correlation Analyses in Experiment 3

\begin{tabular}{lllll}
\hline & Objective delay & Subjective delay & Perceived probability & Discount factor \\
\hline Objective delay & & $2.371 \times 10^{116}[0.727,0.859]$ & $2.504 \times 10^{80}[-0.730,-0.569]$ & $8.457 \times 10^{68}[-0.682,-0.548]$ \\
Subjective delay & $1.498 \times 10^{104}[0.685,0.818]$ & & $1.083 \times 10^{74}[-0.703,-0.570]$ & $7.391 \times 10^{65}[-0.668,-0.534]$ \\
Perceived probability & $5.096 \times 10^{62}[-0.654,-0.520]$ & $8.837 \times 10^{56}[-0.627,-0.493]$ & & $8.987 \times 10^{77}[0.586,0.719]$ \\
Discount factor & $1.060 \times 10^{52}[-0.603,-0.469]$ & $3.706 \times 10^{44}[-0.564,-0.430]$ & $6.619 \times 10^{58}[0.501,0.635]$ & \\
\hline
\end{tabular}

Note. Each cell shows the Bayes factor in favor of the alternative hypothesis and the 95\% CI of the Kendall correlation coefficient (in brackets). The results of the long-perception group are shown above the diagonal whereas those of the short-perception group are shown below the diagonal

Figs. 5 and 6 for the relevant scatterplots. All but two participants showed the same pattern of associations at an individual level (see Fig. 7). Finally, Bayesian path analyses revealed evidence for both the direct effect from objective delay to risk perception and the indirect effect via time perception. See Table 8 for the point estimates and 95\% CIs of the standardized path coefficients.

\section{Discussion}

By experimentally manipulating time perception using the date/delay effect, this study revealed a causal impact of time perception on risk perception and delay discounting. Specifically, longer perceptions of the same set of objective delays led to higher levels of perceived risk and in turn higher levels of delay discounting. This suggests that the impact of objective delay on risk perception and delay discounting is mediated by time perception of objective delay. The results of Bayesian path analyses and relevant group- and individuallevel correlation analyses also suggested that time perception partially mediated the effect of objective delay on risk perception. Finally, correlation analyses demonstrated the same connections among objective delay, perceived probability, and discount factor at both group and individual levels, further corroborating the results of the first two experiments.

\section{General discussion}

Psychological mechanisms underlying delay discounting have attracted much attention from researchers interested in intertemporal choice. Several previous studies suggesting critical roles of time and risk perceptions in delay discounting relied on only correlational data. To improve understanding of the relationships among time perception, risk perception, and delay discounting, this research examined the possible
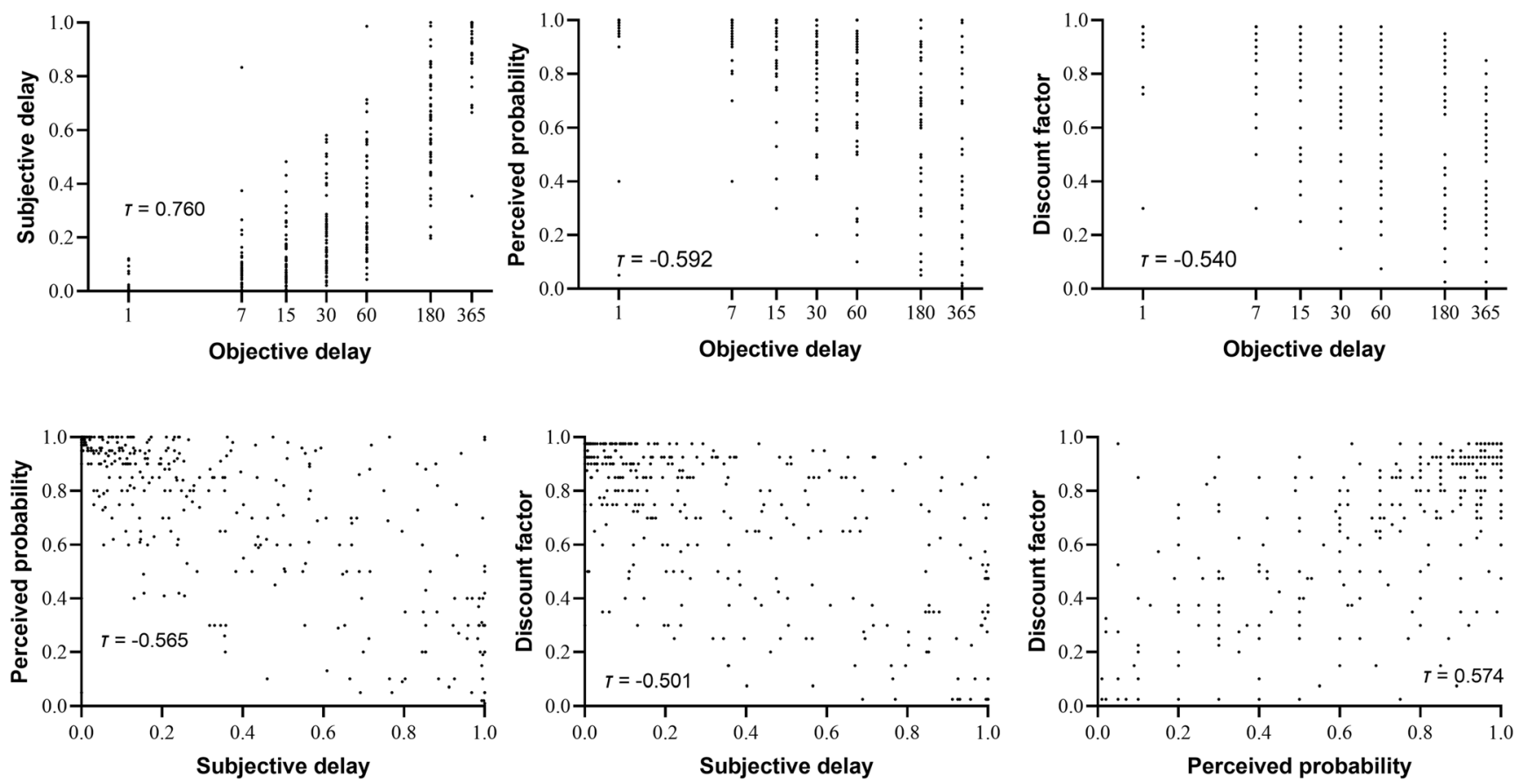

Fig. 5 Scatterplots for the group-level associations among objective delay, subjective delay, perceived probability, and discount factor for the shortperception group in Experiment 3. The axis for objective delay was logarithmic-transformed to express the values more clearly 

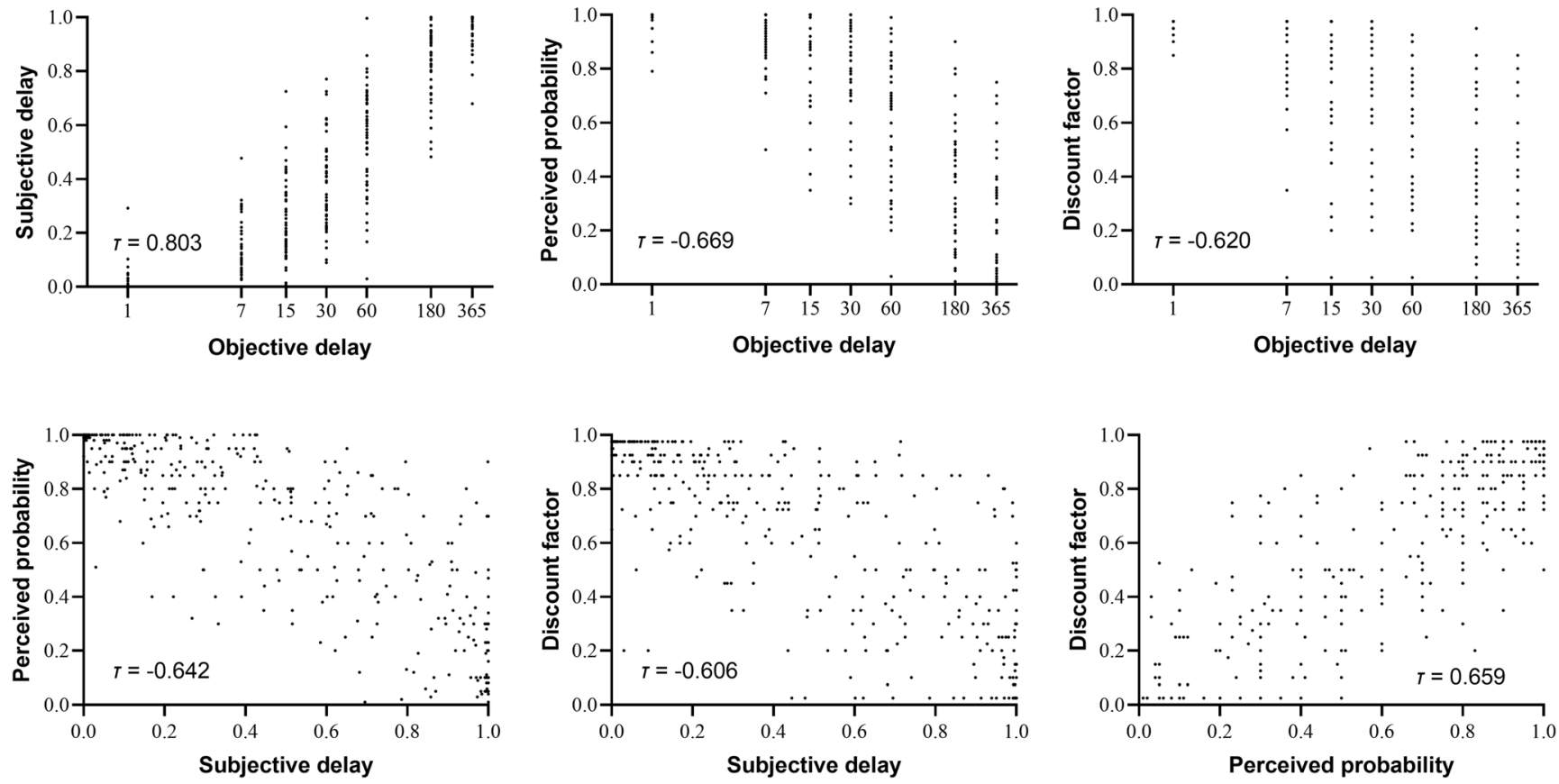

Fig. 6 Scatterplots for the group-level associations among objective delay, subjective delay, perceived probability, and discount factor for the longperception group in Experiment 3. The axis for objective delay was logarithmic-transformed to express the values more clearly

causal impacts of time and risk perceptions on delay discounting with three experiments that externally manipulated objective delay, time perception, and risk perception. With such manipulations, Experiment 1 showed that longer objective delays caused higher levels of risk perception and delay discounting; Experiment 2 found that higher levels of risk
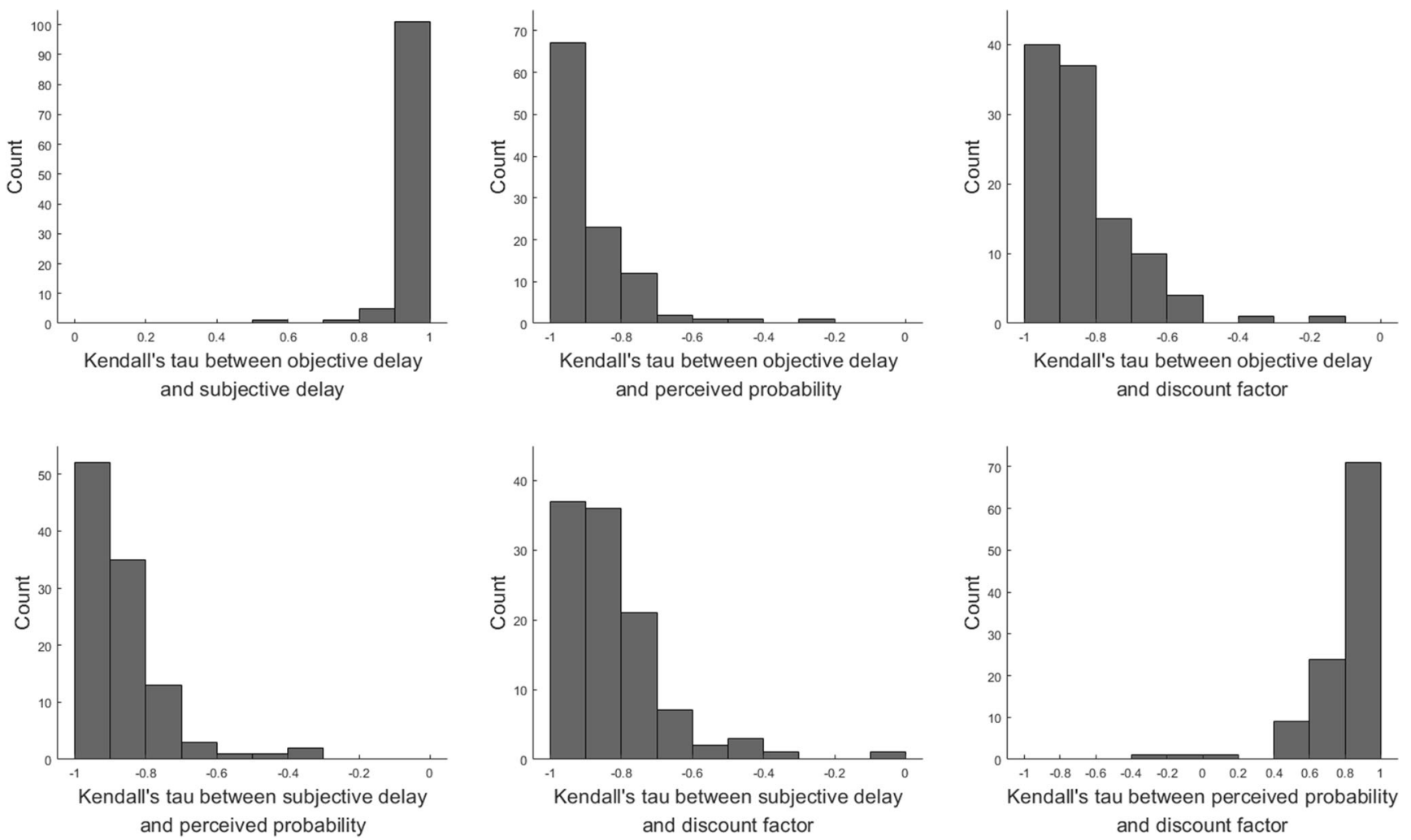

Fig. 7 Distributions of individual Kendall's tau for the associations among objective delay, subjective delay, perceived probability, and discount factor in Experiment 3 
Table 8 Point estimates and 95\% CIs (in brackets) of the path coefficients from the Bayesian path analyses regarding the direct and indirect (via time perception) effects of objective delay on risk perception in Experiment 3

\begin{tabular}{llll}
\hline Effect & Short-perception group & Long-perception group & Across \\
\hline Direct & $-0.211[-0.355,-0.065]$ & $-0.283[-0.385,-0.180]$ & $-0.241[-0.322,-0.156]$ \\
Indirect & $-0.483[-0.615,-0.352]$ & $-0.485[-0.578,-0.395]$ & $-0.488[-0.563,-0.417]$ \\
\hline
\end{tabular}

perception led to higher levels of delay discounting; and Experiment 3 showed that time perception causally mediated the influence of objective delay on risk perception. Overall, the results suggested a causal pathway from objective delay to delay discounting through time and risk perceptions.

Several researchers have attempted to build a connection between risky and intertemporal decisions (e.g., Mischel \& Grusec, 1967; Rachlin, Logue, Gibbon, \& Frankel, 1986). Both types of decision can be understood from a discounting perspective in that when an outcome is coupled with delay or risk, its value would be discounted. Additionally, many behavioral effects in risky and intertemporal decisions can be viewed as counterparts between the two domains (e.g., Prelec \& Loewenstein, 1991). For example, the common ratio effect in risky choice shares the same behavioral pattern as the common difference effect in intertemporal choice. Such similarities have led some researchers to propose a common psychological mechanism for both risky and intertemporal decisions. Some scholars argued that probability discounting was a major contributor to delay discounting since anything delayed is associated with risk - that is, the implicit-risk hypothesis (also known as the delay-as-risk hypothesis; e.g., Benzion, Rapoport, \& Yagil, 1989; Mischel \& Grusec, 1967). Other scholars believed that delay discounting is the underlying reason for probability discounting since anything risky might not happen immediately but could be harvested only after a random delay produced by repeated attempts, that is, the risk-as-delay hypothesis (e.g., Ostaszewski, Green, \& Myerson, 1998; Rachlin et al., 1986).

Most studies regarding the common mechanism underlying risky and intertemporal decisions have focused on the implicit risk or delay-as-risk hypothesis (e.g., Bixter \& Luhmann, 2015; Keren \& Roelofsma, 1995; Weber \& Chapman, 2005). Therefore, in this investigation we tried to establish a causal link from the implicit risk involved in a delayed outcome to the explicit delay-discounting phenomenon. By using more appropriate instruction for the riskperception task and measuring risk perception and delay discounting in a proper order, Experiment 1 found that levels of risk perception and delay discounting were positively correlated. Moreover, by directly manipulating risk perception in Experiment 2, the correlational relationship was shown to be causal in nature. Therefore, the current studies offered a critical piece of evidence for the implicit-risk hypothesis. On the other hand, the empirical support for the risk-as-delay hypothesis mainly came from the relatively good performance of the relevant models in fitting behavioral data from risky choice tasks (Ostaszewski, Green, \& Myerson, 1998; Rachlin, Raineri, \& Cross, 1991; Yi, de la Piedad, \& Bickel, 2006). To the best of our knowledge, no direct test of the riskas-delay hypothesis has been conducted. Future research should explore the causal relationship between risk and delay in a way similar to ours to further compare the risk-as-delay and delay-as-risk hypotheses.

Acknowledgements This research was supported by National Nature Science Foundation of China to Junyi Dai (Grant No. 31872780) and the Fundamental Research Funds for the Central Universities of China to Junyi Dai (Grant No. 2018QNA3014).

\section{Appendix}

The titration procedure for adjusting the amount of the immediate reward in the delay-discounting task

For each delay, the amount of the delayed reward was fixed at $40 \mathrm{CNY}$ (Chinese Yuan) and two adjustment bounds were set for the immediate amount, with the initial upper bound (UB) equal to the delayed amount (i.e., $40 \mathrm{CNY}$ ) and the initial lower bound (LB) fixed at 0 CNY. The immediate amount was always equal to the average of the two adjustment bounds, leading to an initial immediate reward of $20 \mathrm{CNY}$. After each choice, the UB would be updated to the average of the UB after the previous trial and the immediate amount for the current trial if the immediate reward was chosen, or the LB would be updated to the average of the LB after the previous trial and the immediate amount of the current trial if the delayed reward was chosen. In either case, the immediate amount would be updated to the average of the two adjustment bounds as in the first trial. For each delay, the titration procedure would stop, and the updated immediate amount would be treated as the indifference point if the two bounds were sufficiently close to each other (i.e., the distance between the bounds was no more than $2 \mathrm{CNY}$ ). Unlike the common adjustment method in which the LB or UB was updated to the current immediate amount after the respective choice response, the titration procedure used in the current studies was more lenient and thus allowed for probabilistic choice responses which had enjoyed substantial support from 
empirical studies (e.g., Dai \& Busemeyer, 2014). Consequently, the indifference points revealed by this titration procedure were more likely to approach the "true" indifference points whose choice probabilities against the delayed reward were $50 \%$.

\section{References}

Benzion, U., Rapoport, A., \& Yagil, J. (1989). Discount rates inferred from decisions: an experimental study. Management Science, 35(3), 270-284.

Bixter, M. T., \& Luhmann, C. C. (2015). Evidence for implicit risk: delay facilitates the processing of uncertainty. Journal of Behavioral Decision Making, 28(4), 347-359.

Blackburn, M., \& El-Deredy, W. (2013). The future is risky: discounting of delayed and uncertain outcomes. Behavioural Processes, 94, 9 18.

Dai, J., \& Busemeyer, J. R. (2014). A probabilistic, dynamic, and attribute-wise model of intertemporal choice. Journal of Experimental Psychology: General. 143, 1489-1514.

Dai, J., Pachur, T., Pleskac, T., \& Hertwig, R. (2019). Tomorrow never knows: Why and how uncertainty matters in intertemporal choice. In R. Hertwig, T. J. Pleskac, T. Pachur \& the Center for Adaptive Rationality, Taming Uncertainty. Cambridge: MIT.

DeHart, W. B., \& Odum, A. L. (2015). The effects of the framing of time on delay discounting. Journal of the Experimental Analysis of Behavior, 103, 10-21.

Du, W., Green, L., \& Myerson, J. (2002). Cross-cultural comparisons of discounting delayed and probabilistic rewards. The Psychological Record, 52(4), 479-492.

Epper, T., Fehr-Duda, H., \& Bruhin, A. (2011). Viewing the future through a warped lens: why uncertainty generates hyperbolic discounting. Journal of Risk \& Uncertainty, 43(3), 169-203.

Frederick, S., Loewenstein, G., \& O'Donoghue, T. (2002). Time discounting and time preference: a critical review. Journal of Economic Literature, 40(2), 351-401.

JASP Team. (2019). JASP (Version 0.10.2)[Computer software].

Jeffery, H. (1961). Theory of probability. Oxford, UK: Oxford University Press.

Johnson, K. L., Bixter, M. T., \& Luhmann, C. C. (2020). Delay discounting and risky choice: Meta-analytic evidence regarding single-process theories. Judgment and Decision Making, 15(3), $381-400$.

Kagel, J. H., Green, L., \& Caraco, T. (1986). When foragers discount the future: constraint or adaptation?. Animal Behaviour, 34, 271-283.

Keren, G., \& Roelofsma, P. (1995). Immediacy and certainty in intertemporal choice. Organizational Behavior and Human Decision Processes, 63(3), 287-297.

Kruschke, J. K., \& Liddell, T. M. (2018). Bayesian data analysis for newcomers. Psychonomic Bulletin \& Review, 25(1), 155-177.

LeBoeuf, R. A. (2006). Discount rates for time versus dates: the sensitivity of discounting to time-interval description. Journal of Marketing Research, 43(1), 59-72.

Lindley, D. V. (1965). Introduction to probability and statistics from a Bayesian point of view, part 2: inference. Cambridge: Cambridge University Press.
Mischel, W., \& Grusec, J. (1967). Waiting for rewards and punishments: Effects of time and probability on choice. Journal of Personality and Social Psychology, 5, 24-31.

Muthén, L. K., \& Muthén, B. O. (1998-2017). Mplus User's Guide: Eighth Edition. Los Angeles, CA: Muthén \& Muthén.

Myerson, J., Green, L., \& Warusawitharana, M. (2001). Area under the curve as a measure of discounting. Journal of the Experimental Analysis of Behavior, 76(2), 235-243.

Ostaszewski, P., Green, L., \& Myerson, J. (1998). Effects of inflation on the subjective value of delayed and probabilistic rewards. Psychonomic Bulletin \& Review, 5(2), 324-333.

Patak, M., \& Reynolds, B. (2007). Question-based assessments of delay discounting: do respondents spontaneously incorporate uncertainty into their valuations for delayed rewards? Addictive Behaviors, 32(2), 0-357.

Prelec, D., \& Loewenstein, G. (1991). Decision making over time and under uncertainty: A common approach. Management science, 37(7), 770-786.

Rachlin, H., Logue, A. W., Gibbon, J., \& Frankel, M. (1986). Cognition and behavior in studies of choice. Psychological Review, 93(1), 3345 .

Rachlin, H. C., Raineri, A., \& Cross, D. V. (1991). Perceived probability and delay. Journal of the Experimental Analysis of Behavior, 55(2), 233-244.

Rae, J. (1834). The Sociological Theory of Capital (reprint 1834 ed.). London: Macmillan.

Read, D. (2004). Intertemporal choice. In D. J. Koehler \& N. Harvey (Eds.), Blackwell Handbook of Judgment and Decision Making (pp. 3-19). Oxford: Blackwell Publishing.

Read, D., Frederick, S., Orsel, B., \& Rahman, J. (2005). Four score and seven years from now: the date/delay effect in temporal discounting. Management Science, 51(9), 1326-1335.

Reynolds, B., Patak, M., \& Shroff, P. (2007). Adolescent smokers rate delayed rewards as less certain than adolescent nonsmokers. Drug and Alcohol Dependence, 90, 301-303.

Rouder, J. N., Speckman, P. L., Sun, D., \& Morey, R. D. (2009). Bayesian $t$ tests for accepting and rejecting the null hypothesis. Psychonomic Bulletin \& Review, 16, 225-237.

Rung, J. M., Argyle, T. M., Siri, J. L., \& Madden, G. J. (2018). Choosing the right delay-discounting task: Completion times and rates of nonsystematic data. Behavioural Processes, 151, 119-125.

Saito, K. (2009). A relationship between risk and time preferences (No. 1477). Discussion Paper, Center for Mathematical Studies in Economics and Management Science.

Sozou, P. D. (1998). On hyperbolic discounting and uncertain hazard rates. Proceedings of the Royal Society of London. Series B: Biological Sciences, 265(1409), 2015-2020.

Stephens, D. W. (2002). Discrimination, discounting and impulsivity: a role for an informational constraint. Philosophical Transactions of the Royal Society of London. Series B: Biological Sciences, 357, $1527-1537$.

Stevenson, M. K. (1986). A discounting model for decisions with delayed positive or negative outcomes. Journal of Experimental Psychology: General, 115, 131-154.

Takahashi, T., Ikeda, K., \& Hasegawa, T. (2007). A hyperbolic decay of subjective probability of obtaining delayed rewards. Behavioral and Brain Functions, 3(1), 52.

Weber, B. J., \& Chapman, G. B. (2005). The combined effects of risk and time on choice: does uncertainty eliminate the immediacy effect? Does delay eliminate the certainty effect? Organizational Behavior and Human Decision Processes, 96(2), 104-118. 
Weber, B. J., \& Huettel, S. A. (2008). The neural substrates of probabilistic and intertemporal decision making. Brain Research, 1234, 104-115.

Wendt, S., \& Czaczkes, T. J. (2017). Individual ant workers show selfcontrol. Biology Letters, 13(10), 20170450.

Yi, R., de la Piedad, X., \& Bickel, W. K. (2006). The combined effects of delay and probability in discounting. Behavioural Processes, 73(2), 149-155.
Zauberman, G., Kim, B. K., Malkoc, S. A., \& Bettman, J. R. (2009). Discounting time and time discounting: subjective time perception and intertemporal preferences. Journal of Marketing Research, 46(4), 543-556.

Publisher's note Springer Nature remains neutral with regard to jurisdictional claims in published maps and institutional affiliations. 